\title{
Minerals with metal-organic framework structures
}

Tomislav Friscic $^{1}$, Igor Huskic ${ }^{1}$, Sergey Krivovichev ${ }^{2}$, Igor Pekov ${ }^{3}$

${ }^{1}$ Department Of Chemistry, McGill University, Montreal, Canada, ${ }^{2}$ Department of Crystallography, St. Petersburg State University, St. Petersburg, Russian Federation, ${ }^{3}$ Faculty of Geology, Lomonosov Moscow State University, Moscow, Russian Federation E-mail: tomislav.friscic@mcgill.ca

Metal-organic frameworks (MOFs) are an increasingly important family of advanced materials based on open, nanometerscale metal-organic architectures, whose design and synthesis are based on the directed assembly of carefully designed subunits.[1] Whereas the development of MOFs has established them as archetypal examples of artificial, designed advanced materials, we now demonstrate an unexpected link between mineralogy and MOF chemistry. In particular, we report the discovery that the rare organic minerals stepanovite and zhemchuzhnikovite exhibit structures almost identical to those found in well-established magnetic and proton-conducting metal oxalate MOFs.[2] The structures of stepanovite and zhemchuzhnikovite, exhibiting almost nanometer-wide and guest-filled apertures and channels, respectively, change the perspective of MOFs as exclusively artificial materials and represent, so far, unique examples of open framework architectures in organic minerals. This presentation will elaborate the structures of these rare and long known but structurally poorly characterized minerals, as well as address the aspects of their polymorphism. We will also consider potential pathways for the formation of such structures in the laboratory, and possibly even in the natural environment. Of particular importance in that context will be solid-state reactivity based on metal oxides, either through mechanochemistry, or through "accelerated aging" - a process highly akin to geological biomineralization processes known as mineral neogenesis or mineral weathering. [3]

[1] Furukawa, H., Cordova, K. E., O’Keeffe, M. \& Yaghi, O. M. (2013). Science, 341, 1230444.

[2] Huskić, I., Pekov, I., Krivovichev, S. \& Friščić, T. (2016). Science Advances, 2, e1600621

[3] Qi, F., Stein, R. S. \& Friščić, T. (2014). Green Chemistry, 16, 121-132.

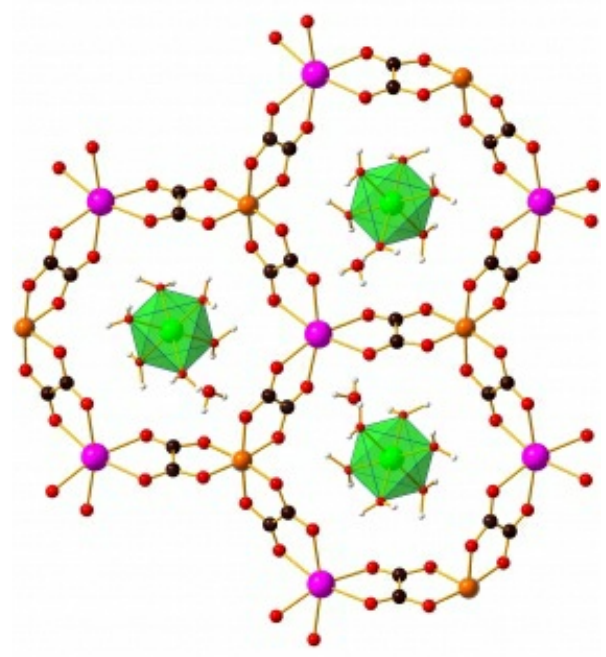

Keywords: Metal-organic frameworks, minerals, crystallography 